\title{
DESIGN OF RASPBERRY PI PARALLEL PROCESSOR
}

\author{
Mohanad ABDULHAMID \\ moh1hamid@yahoo.com \\ Mwalimu MUTHAMI \\ researcher12018@yahoo.com \\ AL-HIKMA UNIVERSITY, BAGHDAD, IRAQ \\ UNIVERSITY OF NAIROBI, KENYA
}

\begin{abstract}
As data centers have increased in size, there has been a need to create clusters out of cheaper, more affordable commodity parts that can easily be replaced upon failure, and that create more affordable data centers overall. However, such large clusters are still outside of feasibility for individuals and small businesses. It is a worthwhile exercise to see if much smaller clusters could be created for such applications, and to compare their performance / price measure to that of the previous traditional data centers. For this paper, such a cluster is created using Raspberry Pis which are small-sized, singleboard computers. A data sharing model is built in Python using message passing interface (MPI) that ran on the cluster of the four Raspberry Pis. So as to evaluate the performance of the system, some greedy algorithms are created. During the implementation process, previously unknown skills, including how to create a cluster, programming the infrastructure are learnt.
\end{abstract}

\section{KEYWORDS:}

Design, raspberry Pi, parallel processor

\section{Introduction}

The demand for much smaller computing devices has always been on the rise. People all over the world want devices which process data faster, are cheaper and readily portable. The current traditional computer models, in desktop and laptop form, are very expensive. This has prompted the production of an alternative computer by use of the Raspberry Pi chips. In addition to being very cheap, one is also free to decide the type of system to be installed. A Raspberry Pi offers access to many general purpose input/outputs (GPIOs) which are not available in many machines. They run on Linux operating system which is more flexible compared to the other operating systems such as windows or Mac OS $\mathrm{X}$ since it is open source. Due to their small sizes, this makes them more portable in comparison to the other computers. 
The reduced size also requires a processor which is fast enough. In order to increase the speed of a typical Raspi computer, many Raspberry Pi chips are connected together in order to share the same load which would have been otherwise computed by a single Raspi computer. Packing several processors into a multi-processor unit to share the load provides a significant increase in computational power. However, the full power of a multi-processor unit will be utilized only if the target software is also designed to utilize multiple parallel processors. In order to utilize multiple processors, a program is designed so that it will divide execution of algorithms into several parallel execution threads that each run simultaneously to each other (also known as parallel programming).

For this paper, a Raspberry Pi parallel processor is designed by connecting four Raspberry Pi chips together, producing a parallel processor which is faster in computing loads when compared to a single Raspberry Pi unit.

For parallel computing, the basic assumption behind it is that a larger problem can be sub-divided into smaller chunks, which can then be operated on each separately at the same time. Parallel computing and related concepts have been used in capital-intensive industries, such as aircraft design and defense for quite a long time. The cost of hardware has dropped over the past few decades combined with the provision of open source operating systems and applications. Individuals with programming passion, students, and some small companies can now use these technologies for their own uses. Traditionally, parallel computing was mainly found within high performance computing (HPC) architectures, such systems being characterized by high speed and high density of calculations. Several researches that discuss the design of Raspberry $\mathrm{Pi}$ cluster can be found in literatures (Cloutier, Paradis \& Weaver, 2016; Jacobus \& Podeschi, 2017; Matthews et. al., 2018; Mitrović, Marković \& Ranđić, 2018; Saffran et al., 2016 ).

\section{Design methodology}

\subsection{Hardware requirements}

\subsubsection{The Raspberry Pi}

There are two Raspberry Pi models: model A and model B. Model A has only one universal serial bus(USB) port, no Ethernet connector and $256 \mathrm{MB}$ memory while model $\mathrm{B}$ has two USB ports and an Ethernet connector and $512 \mathrm{MB}$ of memory. Hence model B is chosen owing to presence of Ethernet connector, more memory and a higher number of USB ports when compared to model A.

The Raspberry Pi's Ethernet port enables connection to a switch. Multiple Raspberry Pis can be connected to a switch forming a cluster. Raspberry Pis use secure digital (SD) cards as secondary storage which allows easy copying of files hence one can create an image of Raspberry Pis operating system which can be readily copied to the other Raspberry Pis. The Raspberry Pi can run the various versions of Linux operating system as well as many software used in implementing clusters and parallel systems.

\subsection{Supplies required to build Raspi supercomputer}

1. 4 Raspberry Pis model B, with 512 MB RAM 4 port

2. USB power hub $(5 \mathrm{v}, 750-1000 \mathrm{Ma})$

USB keyboard

3. One 5-port Ethernet switch

4. 4 Ethernet cables

5. 4 USB cables

\subsubsection{Hardware Setup}

Figure no. 1 shows the schematic diagram of 4-node parallel processor, while, Figure no. 2 shows 4-node Raspberry Pi cluster setup. 


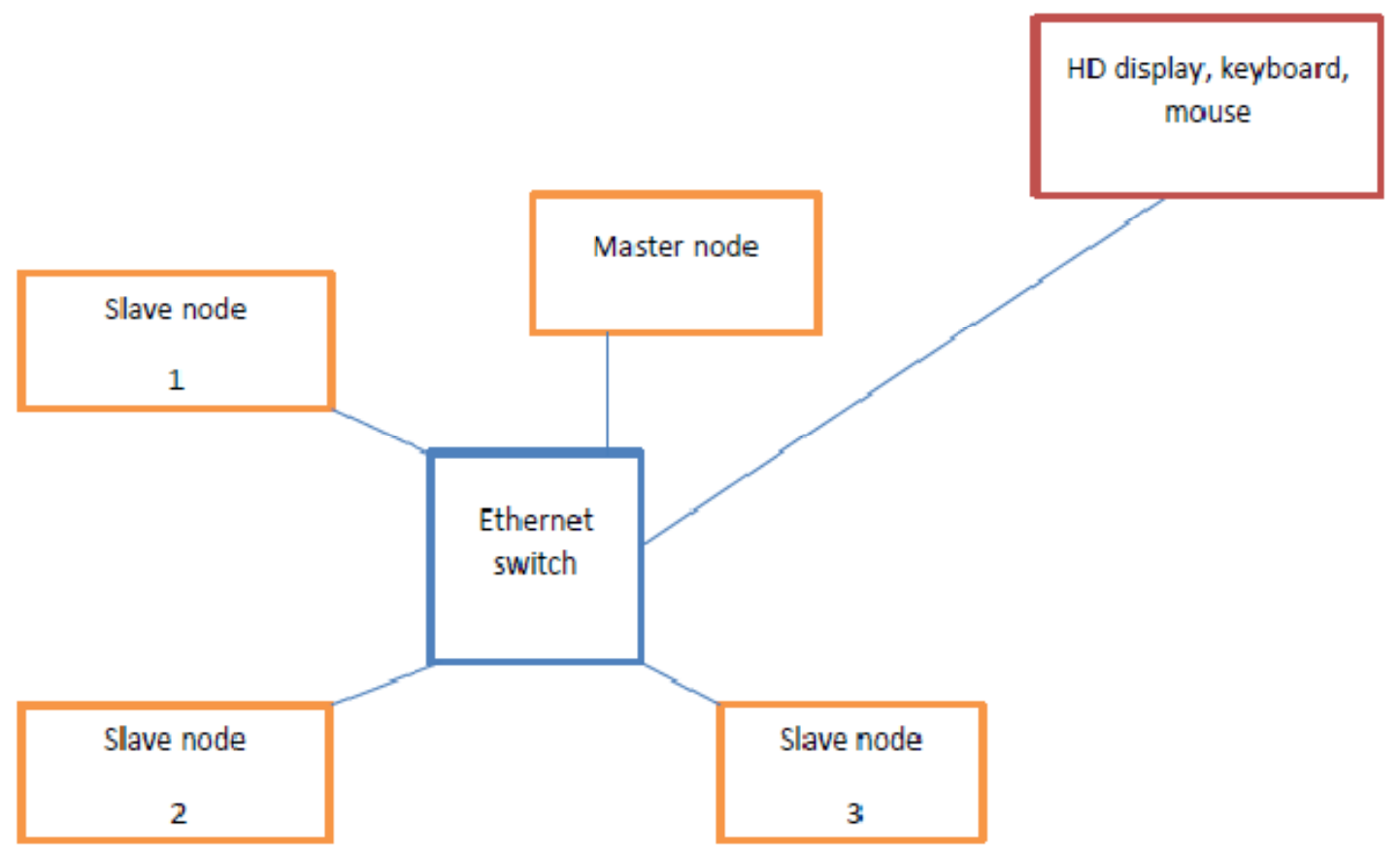

Figure no. 1: 4-node parallel processor

(Source: Jacobus \& Podeschi, 2017)

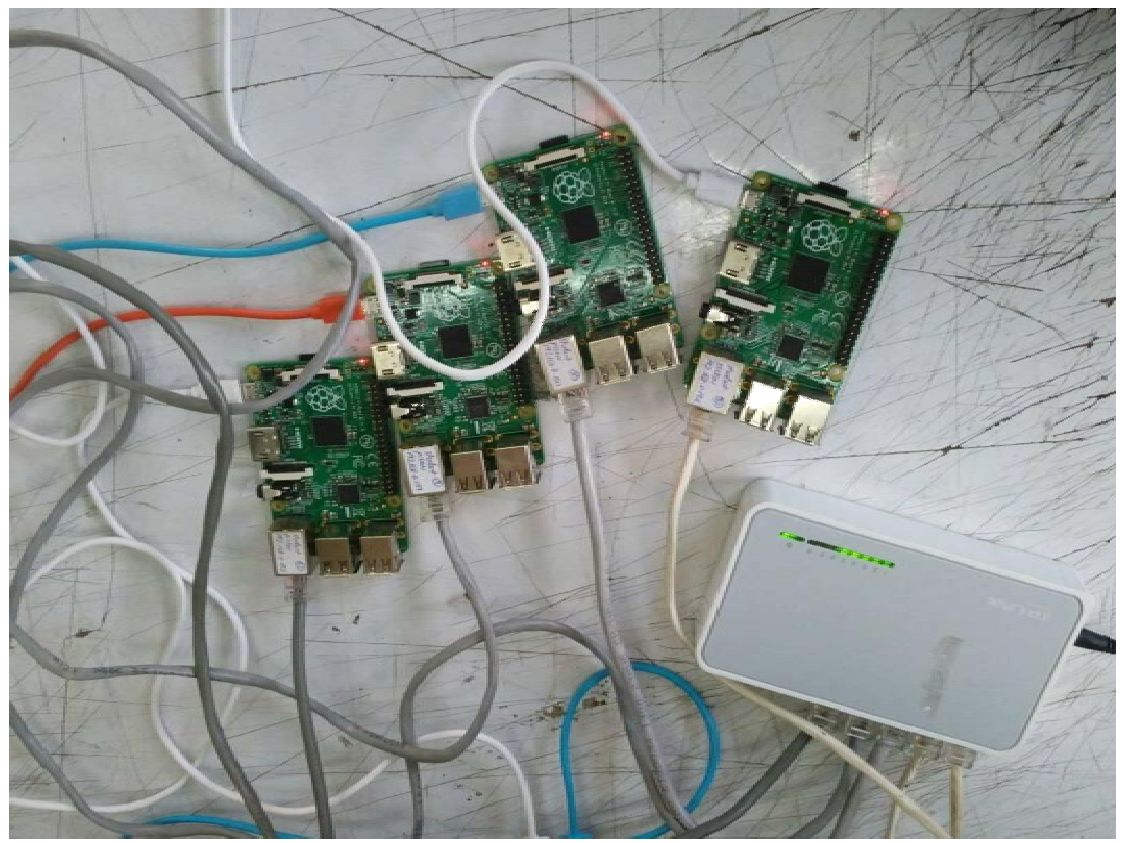

Figure no. 2: 4-node Raspberry Pi cluster setup

(Source: Jacobus \& Podeschi, 2017)

As shown in Figure no. 1, one Raspberry $\mathrm{Pi}$ is selected to act as the master. The keyboard, mouse and the monitor of the personal computer are then connected to that Raspberry Pi. Due to the high number of Raspberry Pis used, a power hub is necessary to power them up. The power hub is connected to a power supply. The Raspberry Pis are then connected to the power hub for powering 
by means of USB cables. The Ethernet switch is connected to the socket rated $240 \mathrm{v}$. The four Raspberry Pis are then connected to the switch ports using Ethernet cables. The function of the switch is to allow inter-Raspberry Pi communication. The switch also acts as a point of access of internet by connecting it to another network using an Ethernet cable. The master node is connected to the PC. The laptop is connected to the switch first to confirm if there is internet connection once the switch has been connected to the internet source.

\subsection{Software setup}

The Raspberry Pi comes equipped with an SD card. The operating system will be run from this card. When compared with an USB hard drive, the SD card is convenient as it is easy to quickly clone for multiple devices, does not take extra desk space and also leaves the USB ports free for connecting the keyboard and the mouse. An SD card is formatted before installing an operating system. Raspbian operating system (OS) is chosen as the OS to be installed on the SD card. Various system configurations are done. Once the installation is complete, the Raspberry $\mathrm{Pi}$ will reboot. PuTTy is installed in the windows. PuTTy is a tool which allows access to the master node and the network through the secure shell (SSH) which is an encrypted secure remote login protocol which uses port 22 by default.

A Raspberry Pi comes installed with a text editor called Nano for editing and running code.

\subsubsection{Booting the Raspberry Pis}

The SD card in the Raspberry Pi is first formatted to delete the stored items present because it had already been used previously. One card is to be cloned to be used on the others. Once formatting is complete, a boot selection screen application is installed. The boot loader installed is new out of box software (NOOBS). A Raspberry Pi operating system (Raspbian wheezy) image is written onto one of the Pi models' SD card. In the Raspberry Pi software configuration tool (raspi-config), the enable boot to desktop option is selected to enable booting to a desktop environment. To allow access to all the latest packages, the following command is run: sudo apt-get update.

\subsubsection{Installing Fortran}

A separate directory for storing Fortran applications is created using the command: mkdir/home/pi/fortran.

Installing Fortran will make the installation of the message passing interface chameleon (MPICH) easier. The command used for installing Fortran is: Sudo apt-get install gfortran

\subsubsection{Installing MPICH3}

The distributed file system is accomplished by the MPICH3 version of the message passing interface (MPI) standard. MPICH3 is chosen because of its ability to operate with many devices. It enables control of multiple processors from one master location by the combined use of cluster architecture and networked nodes. MPICH is an implementation of MPI. The MPI is a communication protocol designed for parallel computing applications. The MPI standard defines a core of routines that can be used by a programmer in order to distribute their application and handle passing back the results of the executed code seamlessly. MPICH3 is more portable between systems when compared to OpenMPI. A directory is created within the home directory where MPICH3 software will be installed. This image is then copied to the rest of the Pi models. The OS allows good integration with hardware and comes loaded with a graphical user interface (GUI) together with development tools. The Raspberry Pi is connected to a computer loaded with PuTTy software using an Ethernet cable. There is difficulty logging into the Raspi using PuTTy from the windows. PuTTy is 
an SSH client used to remotely access and control the Raspberry $\mathrm{Pi}$ running on a windows platform. This is solved by installing xubuntu - a light version of Ubuntu which allowed logging into the Raspis by first getting their internet protocol (IP) addresses.

The Raspi image is now copied to the rest of the SD cards using the Win32Diskimager software ending up with 4 SD cards with the same image. Each individual SD card is plugged into the particular Raspberry Pi in order to change the host name as Pi01, Pi02, Pi03 and Picontroller which is the master node using the following steps by using the command sudo raspi-config:

1. Expand file system

2. Advanced options - hostname Pi01

The above steps are repeated for $\mathrm{Pi} 02$ and $\mathrm{Pi} 03$.

The IP addresses for the different Raspberry Pis are as follows:

Picontroller - 10.42.0.153

Pi01 - 10.42.0.134

$\mathrm{Pi} 02$ - 10.42.0.60

Pi03 - 10.42.0.30

After this, the IP addresses are stored in host file referred to as machine file which would be the file containing the hosts which to start the processes on using nano machinefile

This is the file used by the MPICH3 for the purpose of communicating and sending/receiving messages between various nodes.

\subsubsection{Configuring SSH keys}

In order to allow communication to each Raspberry $\mathrm{Pi}$ without use of passwords, SSH keys are generated for each Raspberry Pi and then each key shared for each device under authorized devices providing a means by which the MPI would be talking to each device without requiring credentials

\subsection{Testing the cluster}

The following command is used to test the cluster

$\begin{array}{lllll}\text { Mpiexec } & -\mathrm{f} & \text { machinefile } & -\mathrm{n} & 4\end{array}$ hostname

Execution of the above command produced the names of the cluster nodes. Hence the MPI is allowing internode communications.

\subsection{How the cluster works}

The program is divided into a number of processes by the master node for the parallel execution. Each process has a reserved region of memory referred to as a stack. Data is added and removed in a last-in-first-out manner in a stack. When a particular function executes, it may add some of its state data to the top of the stack but when the function exits, it removes that data from the stack. A buffer memory system is used to hold the data during the processing. This program is then copied on both nodes. This code is run in the master node. The structure of the program is such that it can be readily be divided between the nodes. The master node sends out the tasks to the slave nodes. Once processing is done, the master node receives the results from the slave nodes, compares them and generates a final result. A scheduler is used to assign as many processes as possible to each node in order to maximize overall performance. It also ensures that events are executed in an orderly manner. 


\subsection{Flow chart for single Pi}

Figure no. 3 shows flow chart for single $\mathrm{Pi}$

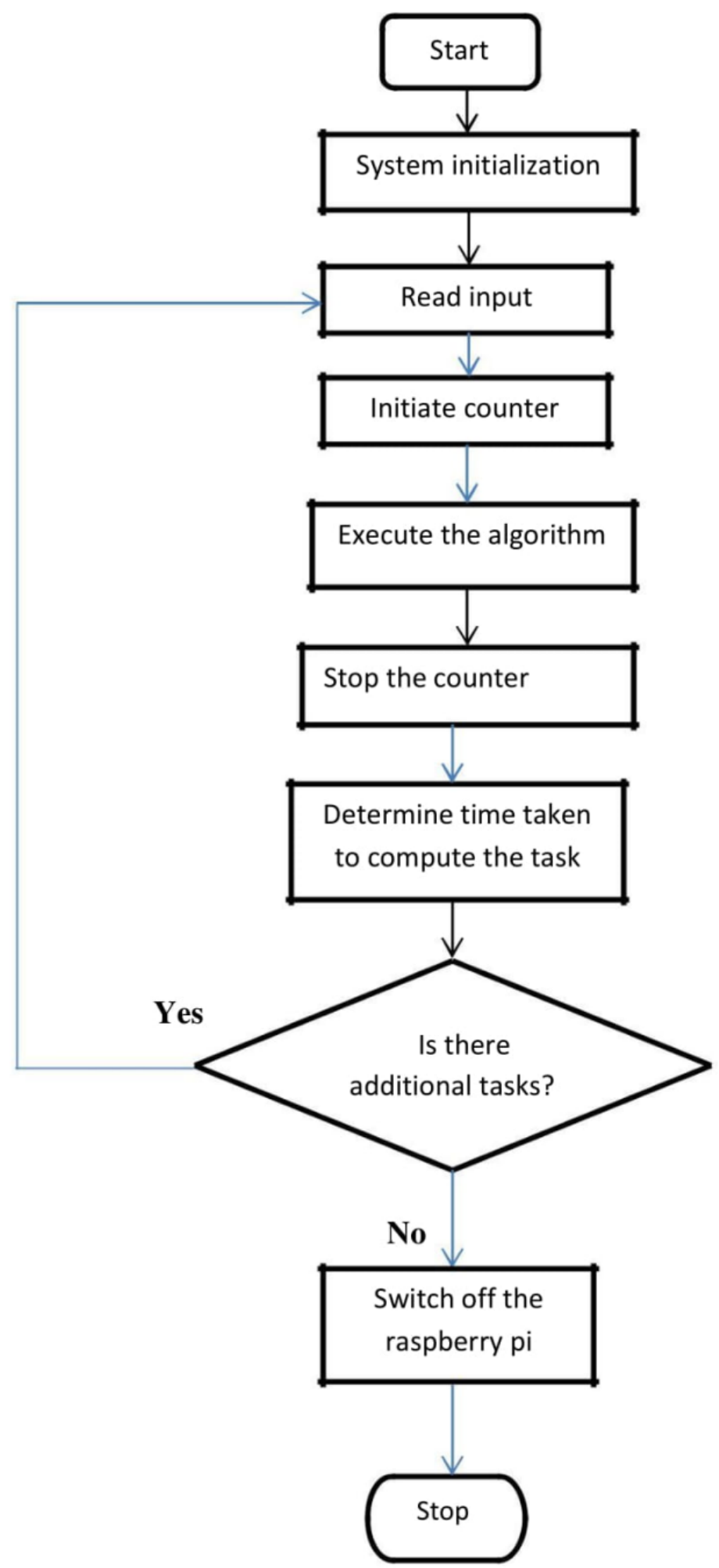

Figure no. 3: Flowchart of single Pi

(Source: Authors) 


\subsection{Flow chart for Raspi parallel processor}

Figure no. 4 shows flow chart for Raspi parallel processor.

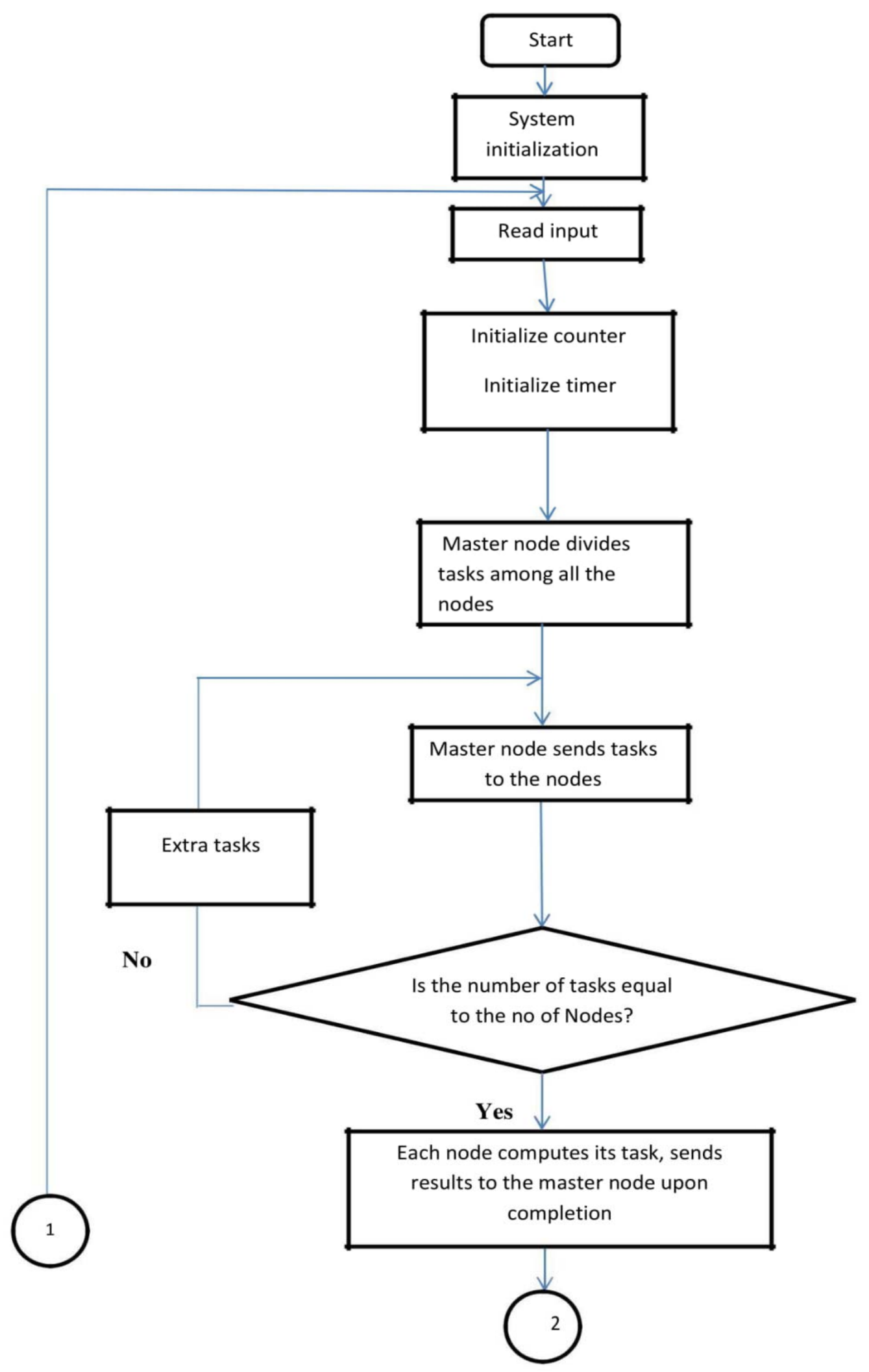




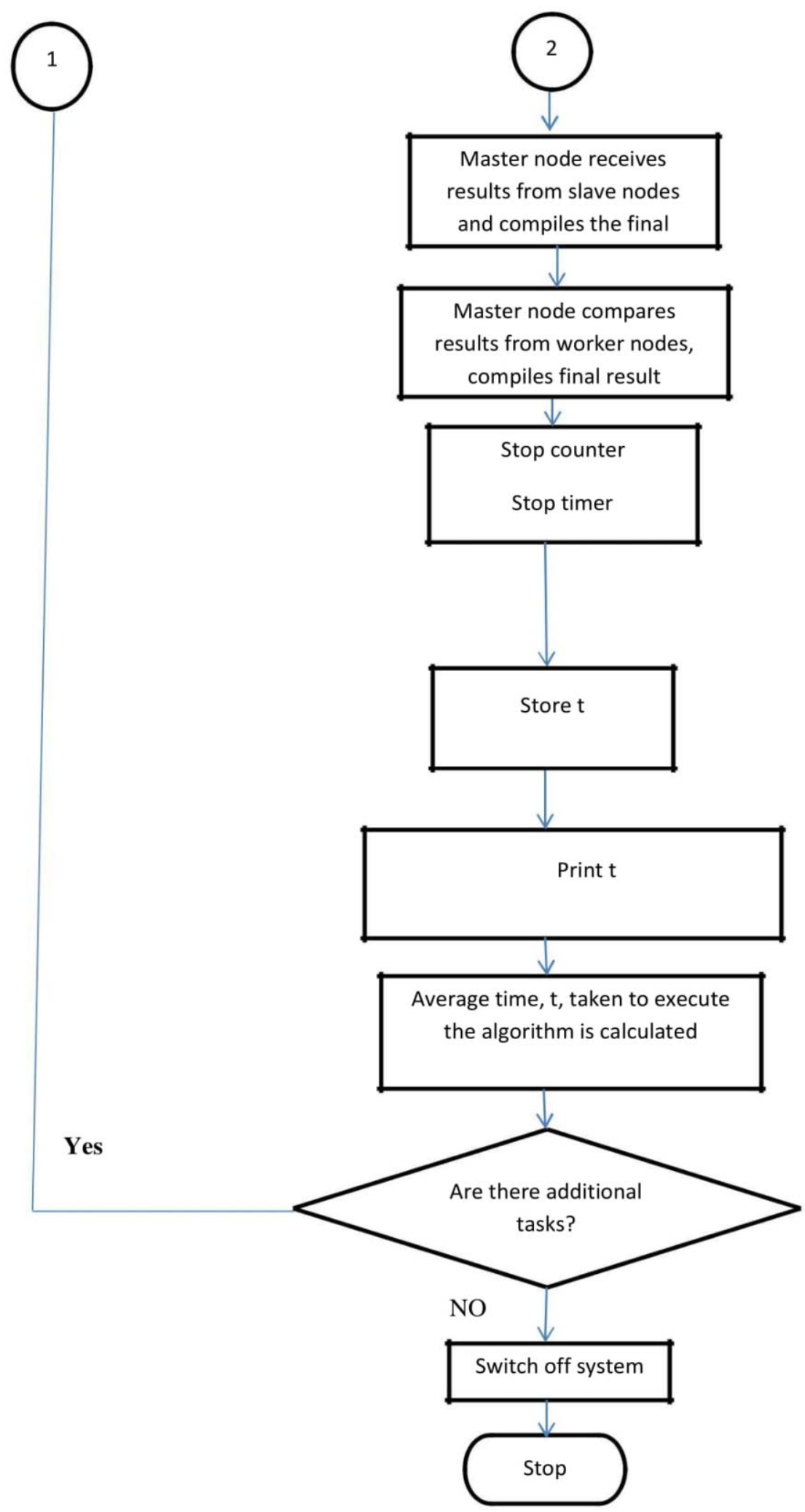

Figure no. 4: Flowchart for Raspi parallel processor

(Source: Authors) 
2.8. Pseudocode for single Raspi

- Start

- Initialize system

- Read input

- Initiate counter

- Run program

- Stop counter

- Determine time taken for execution

- Switch off the Raspberry $\mathrm{Pi}$ if there are no further tasks to be processed

- Stop

\subsection{Pseudocode for the Raspi cluster}

- Start

- Initialize system

- Read the input algorithm

- Distribute tasks from master node to the slave nodes together with python commands

- If the number of tasks is greater than the number of nodes, wait for the nodes to process the currents tasks. After they send their results to the master node, reallocate the remaining tasks

- Each node computes its own task

- Master node receives computation results from each slave node, combines them together to get the final result

- Stop the counter

- Average time taken to complete executing the algorithm is computed

- If there are no more tasks, then the system is switched off, otherwise more input can be entered for another processing

- Stop

\subsection{Greedy algorithms}

The greedy algorithms chosen for this paper are the coin changing problem and the knapsack problem.

\subsubsection{Coin changing problem}

This is a greedy algorithm used to find the lowest/minimum number of coins from a given amount $\mathrm{A}$. The denominations used are ksh 1, ksh 5, ksh 10, ksh 20, ksh 50, ksh 100, ksh 200, ksh 500 and ksh 1000 .

For this part, the total amount chosen from which to get the change is $9,583,786$. In order to use a greedy approach, the greedy choice starts with the highest possible denomination in which our case is ksh 1000, with the other denominations being added as long as the value which remains is greater than zero. Each value of the denomination is subtracted from the total amount $\mathrm{A}$. if the total amount is equal to zero, the result of the various denominations used is printed to the console.

\subsubsection{The knapsack problem}

This greedy algorithm works with the fact that if one is given a set of items with every item having a weight and a value, it is required to find the number of each item to be included within the specified collection so that the total weight is less than or equal to a given limit whereas the total value is as large as possible.

\section{Results}

\subsection{Results for testing cluster}

In order to test for the MPI cluster, the command (Mpiexec $-\mathrm{f}$ machinefile $-\mathrm{n} 4$ hostname) is run in the master node which produced the results below:

- Picontroller

- Pi01

$-\mathrm{Pi02}$

$-\mathrm{Pi} 03$

\subsection{Results for greedy algorithms}

For both the coin changing problem and the knapsack problem, as the number of Raspberry Pis is being increased from one to four the amount of time taken for executing a similar problem is reducing showing inverse relationship as shown in Table no. 1 and Table no. 2 . 
Table no. 1

Results for coin changing problem

\begin{tabular}{|l|l|l|l|l|}
\hline No of pis & 1 & 2 & 3 & 4 \\
\hline $\begin{array}{l}\text { Average } \\
\text { Execution } \\
\text { time(S) }\end{array}$ & 0.124 & 0.085 & 0.077 & 0.0754 \\
\hline
\end{tabular}

(Source: Authors)

Table no. 2

Results for knapsack problem

\begin{tabular}{|l|l|l|l|l|}
\hline No of pis & 1 & 2 & 3 & 4 \\
\hline $\begin{array}{l}\text { Average } \\
\text { Execution } \\
\text { time(S) }\end{array}$ & 0.124 & 0.085 & 0.077 & 0.0754 \\
\hline
\end{tabular}

(Source: Authors)

For the image shown in Figure no. 5, the coin change program is run first on the 3 Pis one at a time with execution taking an average of $0.12 \mathrm{~s}$ for the three slaves (Pi01, Pi02, Pi03). The same code is then executed on the master node (Picontroller) for the whole cluster taking an average of $0.076 \mathrm{~s}$ hence proving that the cluster is more efficient in computing loads.

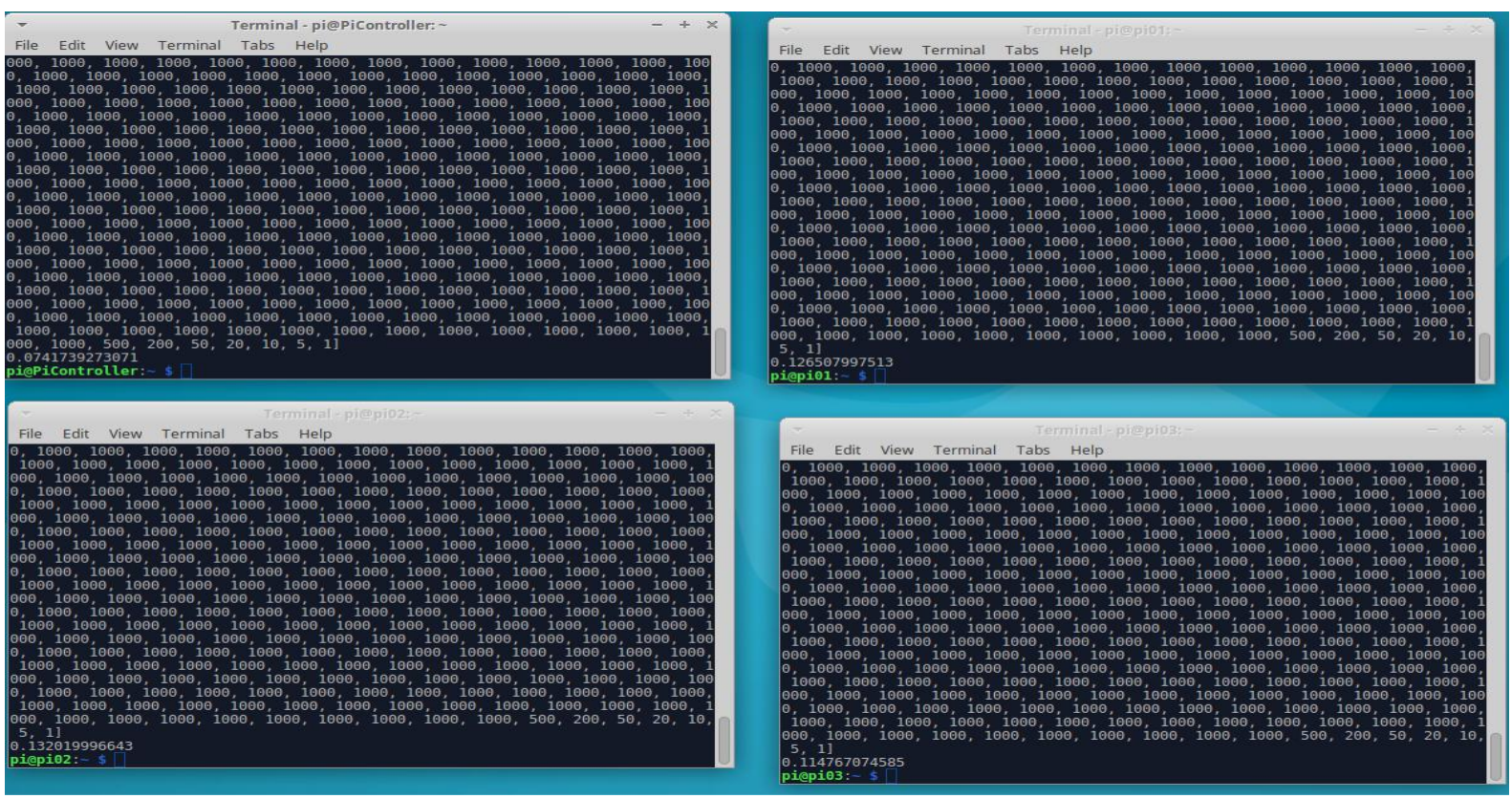

Figure no. 5: Coin changing problem results

(Source: Authors)

The image shown in Figure no. 6 shows program execution results of knapsack problem for all Pis from master node (Picontroller) taking an average of about $0.0041 \mathrm{~s}$. Each slave node (Pi01, Pi02, Pi03) took about 0.0059 s when acting alone. 


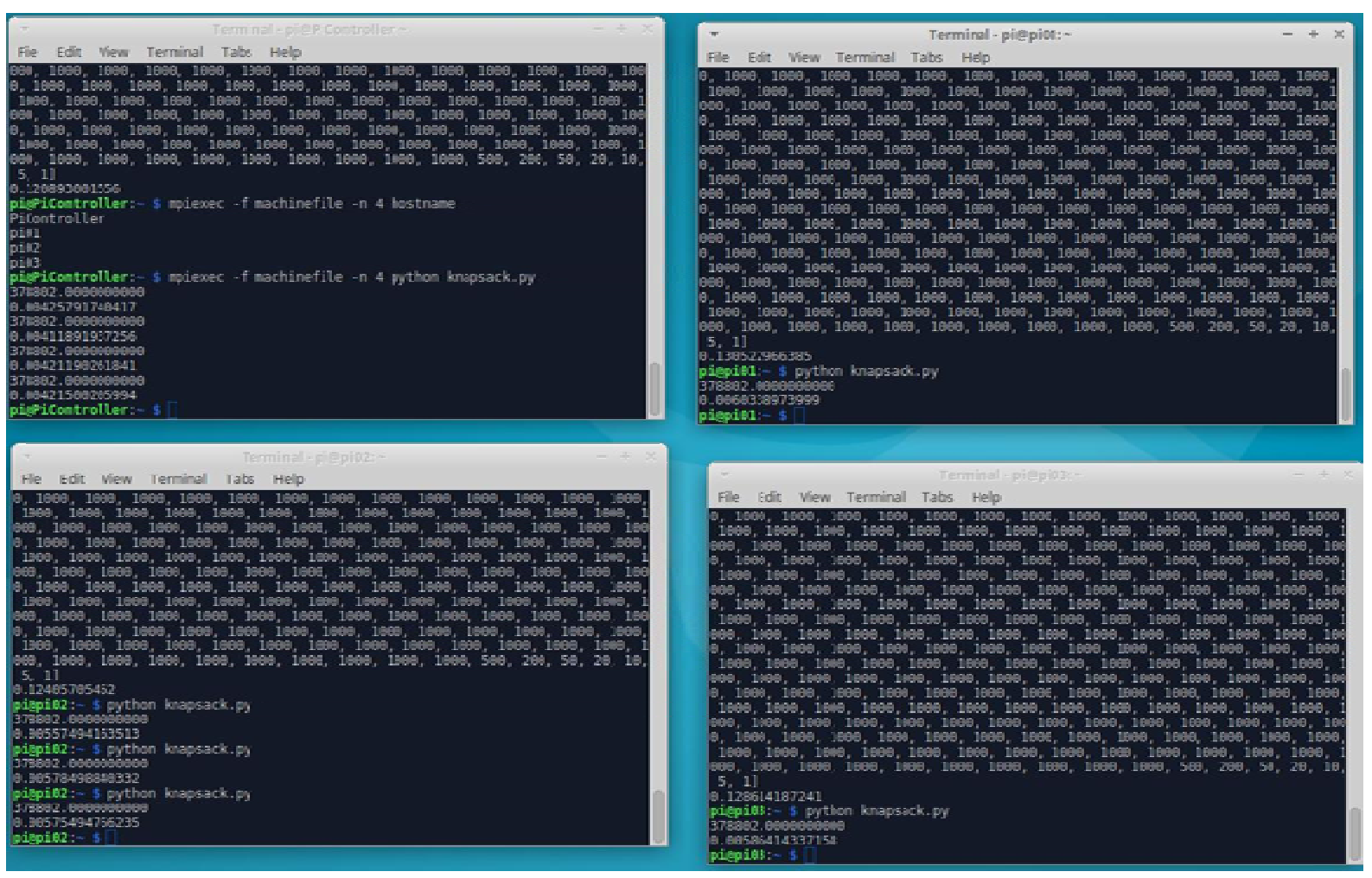

Figure no. 6: Knapsack problem results

(Source: Authors)

\section{Discussion}

As the number of Raspis was being increased from 1 to 4 , the amount of time used to compute a particular program was decreasing accordingly. A higher number of Pis meant that the load was being shared among the nodes, hence consuming a smaller amount of time in executing a particular algorithm. Some Pis were found to be a little faster than the others but the difference was very minimal. This could have resulted in some errors during compiling the amount of time taken to compute a certain task.

Running a computation in multiple processes utilizes some communication between these processes. The communication between various processes takes the form of streams of bytes that travel through specific communication channels. In order to send an object from one process to another, Python has to convert it to a stream of bytes, and then assemble the object back at the receiving end. Python's mechanism for doing these conversions was originally designed for storing objects in files and is implemented in using the pickle module. Every argument that is passed to a Python function running in another process is pickled and then un-pickled. The result of the function undergoes the same process on its way back.

However, most objects can be pickled but some cannot. Second, pickling and un-pickling take time and can sometimes add considerable overhead to your multiprocessing.

For python, functions and classes can only be pickled if they are defined at the top level of a module. This means, for example, that if you define a function inside another function, you cannot pickle it and thus not pass it to a multiprocessing task.

\section{Conclusions}

A Raspberry Pi based parallel processor was designed, tested and found to be working. It was found to share load 
amongst the individual Raspberry Pi units. It also speeded up its computational time as evidenced by the combined unit taking less time when compared to a single
Raspberry Pi. Hence the objective of this which was to design a Raspberry Pi-based processor with the ability to share load was achieved.

\section{REFERENCES}

Cloutier, M., Paradis, C., \& Weaver, V. (2016). A Raspberry Pi cluster instrumented for fine-grained power measurement. Electronics, Vol. 5, No. 4.

Jacobus, B., \& Podeschi, R. (2017). Low-cost cluster computing using Raspberry Pi with mathematica. Proceedings of the EDSIG Conference, USA.

Matthews, S. et al. (2018). Portable parallel computing with the Raspberry Pi. Proceedings of the $49^{\text {th }}$ ACM Technical Symposium on Computer Science Education, USA.

Mitrović, D., Marković, D., \& Ranđić, S. (2018). Raspberry Pi module clustering and cluster application capabilities. $7^{\text {th }}$ International Scientific Conference Technics and Informatics in Education, Serbia.

Saffran, J. et al. (2016). A low-cost energy-efficient Raspberry Pi cluster for data mining algorithms. European Conference on Parallel Processing, France. 